\title{
Observations of diverging field-aligned ion flow with the ESR
}

\author{
S. C. Buchert ${ }^{1,3}$, Y. Ogawa ${ }^{1,}$, R. Fujii ${ }^{1}$, and A. P. van Eyken ${ }^{2}$ \\ ${ }^{1}$ Solar Terrestrial Environment Laboratory, Nagoya University, Nagoya, Japan \\ ${ }^{2}$ EISCAT Scientific Association, Headquarters, Kiruna, Sweden \\ ${ }^{3}$ Now at Swedish Institute of Space Physics, Uppsala, Sweden
}

Received: 28 March 2002 - Revised: 1 July 2003 - Accepted: 13 August 2003 - Published: 19 March 2004

\begin{abstract}
We report on observations of a diverging ion flow along the geomagnetic field that is often seen at the EISCAT Svalbard radar. The flow is upward above the peak of the electron density in the F-region and downward below the peak. We estimate that in such events mass transport along the field line is important for the ionization balance, and that the shape of the F-layer and its ion composition should be strongly influenced by it. Diverging flow typically occurs when there are signatures of direct entry of sheath plasma to the ionosphere in the form of intense soft particle precipitation, and we suggest that it is caused by the ionization and ionospheric electron heating associated with this precipitation. On average, $30 \%$ of all events with ion upflow also show significant ion downflow below.
\end{abstract}

Key words. Ionosphere (polar ionosphere; ionization mechanism; plasma temperature and density)

\section{Introduction}

In the polar regions of the Earth's ionosphere ions can flow along the geomagnetic field and thus, transport plasma from one altitude to another. This transport has influence on the ionization balance, as well as an ion composition and even the composition of the thermosphere. Upward flowing ions can in principle, escape from the earth on open field lines and be transported away by the solar wind. Upflow of ionospheric ions may stand at the beginning of a chain of processes, successively accelerating and energizing particles from the ionosphere and supplying plasma to the magnetosphere. Estimates from more than a decade ago showed that the ionospheric source alone is sufficient to supply the entire magnetospheric plasma content under all geomagnetic conditions (Chappell et al., 1987). After advances in instrumentation and measuring in situ ions at even relatively low energy, these early estimates were essentially confirmed (e.g. Chappell et al., 2000).

In the ionosphere mainly incoherent scatter radars (ISR) have been used to investigate both ion up- and downflow.

Correspondence to: S. C. Buchert

(stephan.buchert@irfu.se)
Wahlund et al. (1992) identified two main processes that are associated with ion upflow, using EISCAT data from Northern Scandinavia. One type is related to periods of strong perpendicular electric fields, enhanced and anisotropic ion temperatures, as well as low electron densities, indicating only small amounts of auroral precipitation. The second type is related to auroral arcs and enhanced electron temperatures. Having ISR data, all terms of the stationary fluid force equation in the direction of the magnetic field can be estimated. Often the forces do not balance according to the observations (Wahlund et al., 1992). Additional mechanisms involving plasma waves that drive ions upward have been postulated, particularly when the second type of upflow related to electron heating by precipitation is effective. A signature of such plasma waves are the so-called naturally enhanced ionacoustic lines in ISR spectra (Rietveld et al., 1991).

At the ISR facility on Svalbard, the ESR (EISCAT Svalbard Radar) is in the cusp/cleft regions during the day. Satellite data have revealed that these regions are sources of topside ionospheric ion outflow. Ionospheric signatures of the cusp were already identified with the Sondrestrom ISR (Watermann et al., 1992), see also (Watermann et al., 1994b), and compared with simulations using observed electron spectra as input parameters (Watermann et al., 1994a). Ion outflow as an additional ionospheric signature of the cusp was first reported by Nilsson et al. (1994). We expect new insight into ionospheric up- and downflow phenomena at relatively low altitudes and energies from the ESR facility. Using combined ESR and EISCAT mainland data Ogawa et al. (2000) find events with strong ion outflow around magnetic local noon associated with enhanced electron density, temperatures, and corresponding to a clear increase in the upward ambipolar force of the ionospheric electrons. However, additional mechanisms that are not accounted for in the standard fluid force balance are suspected to play a role.

Also, ion downflow events are seen at any time and at the geomagnetic disturbance level in EISCAT VHF data (Endo et al., 2000). This downflow is usually isolated from upflow events. Simulations have predicted that ion downflow occurs after ion heating and upwelling, if no further upward ion acceleration at higher altitudes takes place (Loranc and St.Maurice, 1994). Then the ions fall back to lower altitudes 
at later times. This ballistic consideration could explain the downflow observed commonly with the VHF radar.

Here we report on the observation of a different type of ion up- and downflow. It is seen frequently in ESR data and occurs simultaneously on the same field line. It is thus a diverging flow in the magnetic field direction. The upflow is usually above the F-region density peak, and the downflow is below. We present typical events, statistical information on occurrence frequencies, discuss the ionization and force balances, and finally come to conclusions.

\section{Instrumentation}

The data for this study were collected with the EISCAT Svalbard radar (ESR) (Wannberg et al., 1997) which is at 75.19 corrected geomagnetic latitude. Local noon is at 11:04 UT. In order to obtain the field-aligned ion velocity as well as the other plasma parameters that are measured with the incoherent scatter technique (Hagfors, 1997), we use data when the steerable ESR antenna with $32 \mathrm{~m}$ diameter is directed at an elevation of $82^{\circ}$ along the geomagnetic field in the F-region ionosphere. Also used are data from the antenna having a $42 \mathrm{~m}$ diameter which is permanently directed along the geomagnetic field.

The raw, pre-integrated data were post-integrated to time resolutions of 1-4 min depending on the type of antenna control that was used in the experiment. Post-integrated data were analysed by us with the help of the "GUISDAP" software package (Lehtinen and Huuskonen, 1996), to obtain the ion velocity, electron density, and electron and ion temperatures. In some cases the pre-integrated spectra were searched for naturally enhanced ion-acoustic lines (NEIALs) and these data records were then removed before the post-integration, in order to minimize contamination of the incoherent scatter spectra.

\section{Observations of events}

In Fig. 1 we show a typical example of a diverging fieldaligned flow event seen with the ESR 32-m antenna. For this event a total of 14 profiles containing NEIALs were found and removed before post-integration. Starting at about 09:50 UT the electron density is enhanced in the F-region, showing sometimes relatively strong variations in time, for example, around 10:30 UT. A very likely cause of this electron density enhancement is electron precipitation with energies of a few ten to a few $100 \mathrm{eV}$. Electrons of this energy are stopped in the F-region by collisions with atmospheric and ionospheric particles. For a sufficiently large precipitation flux the F-layer is considerably enhanced above the densities in the ambient plasma outside the precipitation region.

We expect that at the same time the ionospheric electrons are heated by the ionization processes and by collisions with precipitating particles. This expectation is confirmed in the panel of Fig. 1 showing $T_{e}$. Above the peak density, at about
$350 \mathrm{~km}$ height, $T_{e}$ is normally almost $4000 \mathrm{~K}$ after 09:50 UT and reaches sometimes values of more than $6000 \mathrm{~K}$.

Also, the ion temperature $T_{i}$ is sometimes increased by Joule heating, for example, at about 10:40 UT. But the ion heating is less strong than the electron heating and occurs only in relatively short periods. In the time of enhanced electron densities most of the thermal energy of the ionosphere is in the electrons.

So far these features of electron and ion density and temperature enhancements are similar to the findings by Nilsson et al. (1994) for the cusp. The panel of Fig. 1 displaying the ion velocity derived from the Doppler shift of the IS spectra as well as velocity profiles displayed in Fig. 2, show that the field-aligned (FA) ion flow is diverging. It is upward in the upper part of the F-region and downward in the lower part. The upward velocity often exceeds $200 \mathrm{~ms}^{-1}$. The downward flow is slower but sometimes clearly reaches about $100 \mathrm{~ms}^{-1}$.

The divergence of the FA flow cannot be explained by a convergence of the perpendicular ion flow. The convection is here not directly observed. But in the F-region to a very good approximation the perpendicular flow $\boldsymbol{v}_{\perp}=\boldsymbol{E} \times \boldsymbol{B} / B^{2}$, where $\boldsymbol{E}$ is the electric field and $\boldsymbol{B}$ is the geomagnetic background field. Since $\boldsymbol{E}$ is electrostatic, $\nabla \times \boldsymbol{E} \approx 0$ and also $\nabla_{\perp} \cdot \boldsymbol{v}_{\perp} \approx 0$, or the perpendicular flow is purely rotational. Therefore, the diverging FA flow must be fed by ongoing ionization, or it removes the F-layer within a time scale of a few minutes. As we have discussed above, the electron density and temperature data indicate that soft precipitation does cause ionization over most of the event shown in Fig. 1.

A one-dimensional continuity equation,

$\frac{\partial}{\partial t} N_{e}+\frac{\partial}{\partial s}\left(v_{i} N_{e}\right)=Q-L$,

describes the ionization balance for our observations approximately, where $N_{e}$ is the electron density, $v_{i}$ is the fieldaligned ion velocity, $s$ is a spatial coordinate along the magnetic field, $Q$ is the ionization production, and $L$ is the ionization loss by chemical reactions and recombination.

Equation (1) is for a flux tube drifting with the convection. However, snapshots are seen by the ESR of flux tubes that pass through the antenna beam. There is no information available about the history of a flux tube seen during one integration period, except when the convection is zero or very small $\left(\lesssim 20 \mathrm{~ms}^{-1}\right)$. Only then are the observed time variations of $N_{e}$ accounted for by the term $\partial N_{e} / \partial t$ in Eq. (1).

In the lower F-region ionization equilibrium according to

$\frac{\partial}{\partial s}\left(F_{i}\right)=Q-L$,

where $F_{i}=N_{e} v_{i}$ is the ion flux, is reached rapidly after any time variations, and the unknown history of the observed flux tube is not a significant problem for the interpretation of our data. However, in the upper F-region it takes much longer to reach ionization equilibrium, and Eq. (2) needs to be applied cautiously. For the event in Fig. 1 enhancements of $T_{i}$ by frictional heating indicate that convection is typically not 


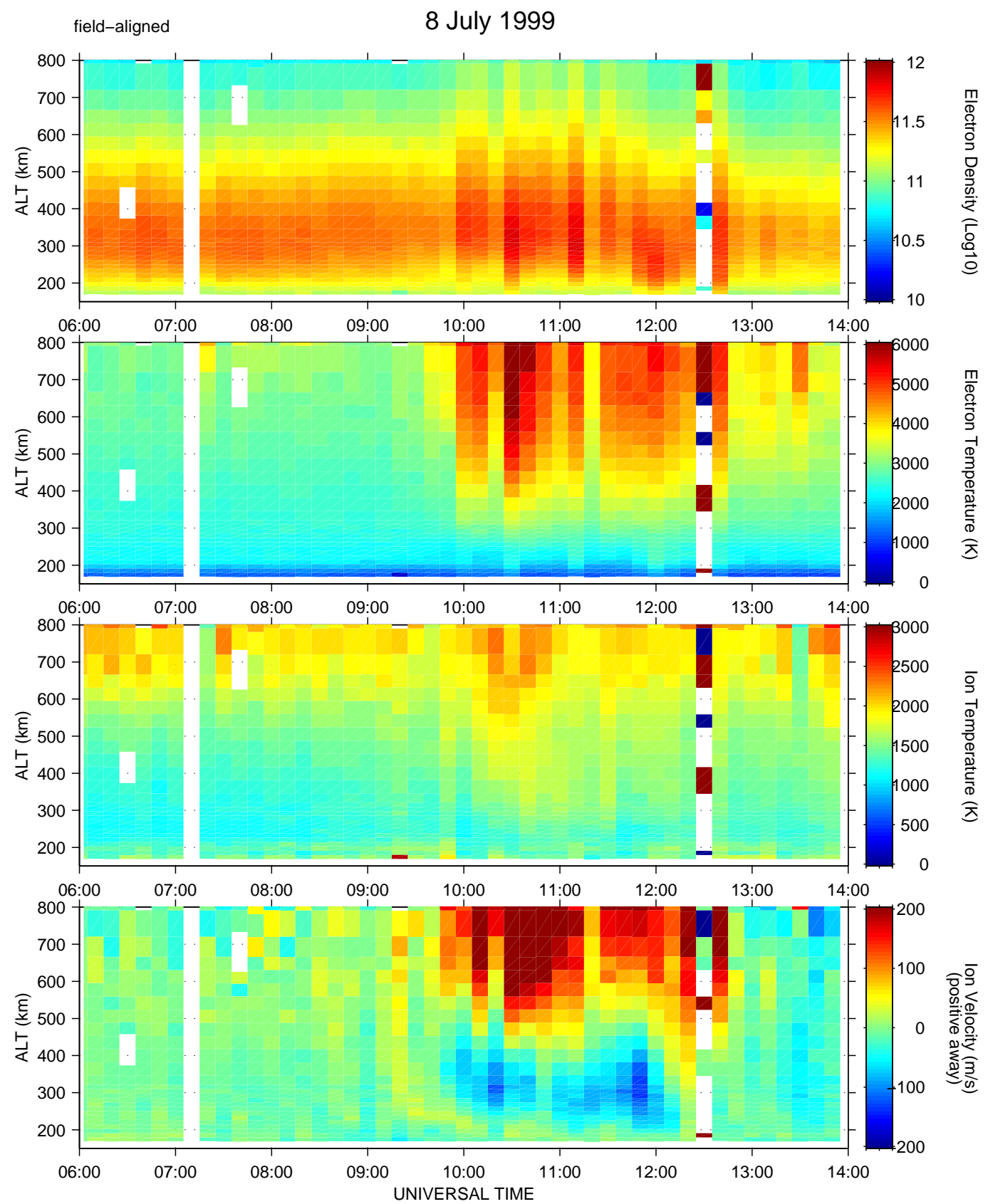

Fig. 1. Overview plot of ESR data from 08 July 1999, 06:00-14:00 UT. Panels from top to bottom show electron density, electron temperature, ion temperature, and ion velocity color-coded over altitude (y-axis) and time (x-axis). The velocity is red for flow away from the radar (upward) and blue for flow toward the radar (downward). Plotted are only data from the field-aligned position of the ESR antenna doing a scan cycle over 4 positions (Common Progamme 2). The data from the field-aligned position are extrapolated to times when the antenna is not field-aligned.

very small. Nevertheless, we proceed with the discussion of the ion flux $F_{i}$, neglecting the term $\partial N_{e} / \partial t$ and keeping in mind the limitations.

Figure 3 shows the flux $F_{i}=N_{e} v_{i}$ calculated from the electron density $N_{e}$ and the ion velocity $v_{i}$ for one of the profiles in Fig. 2. Between about 310 and $550 \mathrm{~km}$ altitude $\partial F_{i} / \partial s>0$, which means that the F-region ionization in this height region is transported away. Consequently, either $\partial N_{e} / \partial t<0$, or there must be ionization sources $Q>L$ in this height re- gion, presumably a combination of ionizing solar radiation and precipitating particles. Below about $310 \mathrm{~km}$, where recombination is dissociative and fast, we have $\partial F_{i} / \partial s<0$. Recombination "eats up" the ions that are transported downward by field-aligned flow. Profiles of the terms in Eq. (2) corresponding to Fig. 3 are plotted in Fig. 4. The transport term $\partial F_{i} / \partial s$ is calculated directly from the observations. The recombination $L$ is estimated from a simple model using the measured $N_{e}$, neutral densities of the MSISE90 

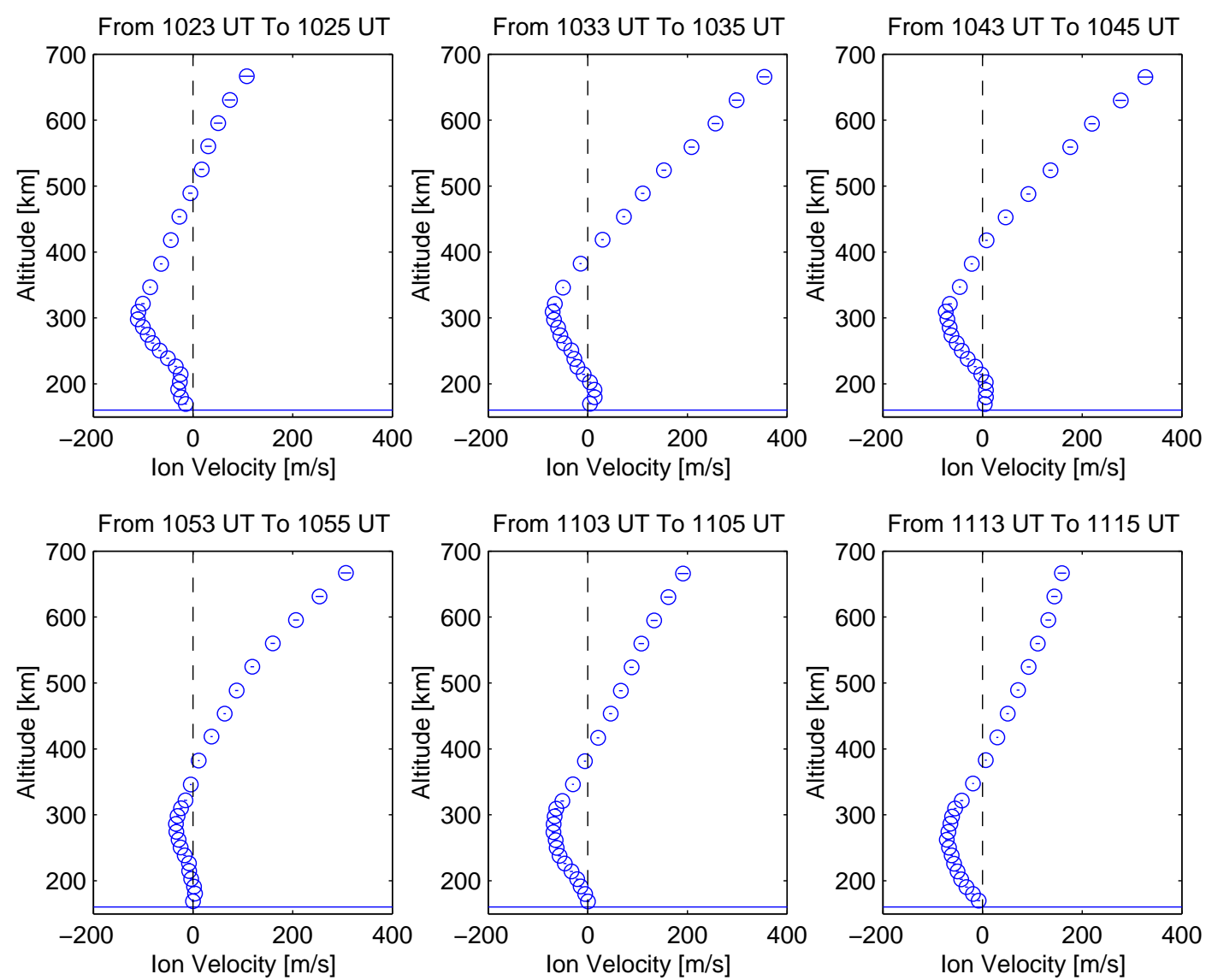

Fig. 2. Profiles of the field-aligned ion velocity observed by the ESR on 08 July 1999, from 10:23 until 11:15 UT.

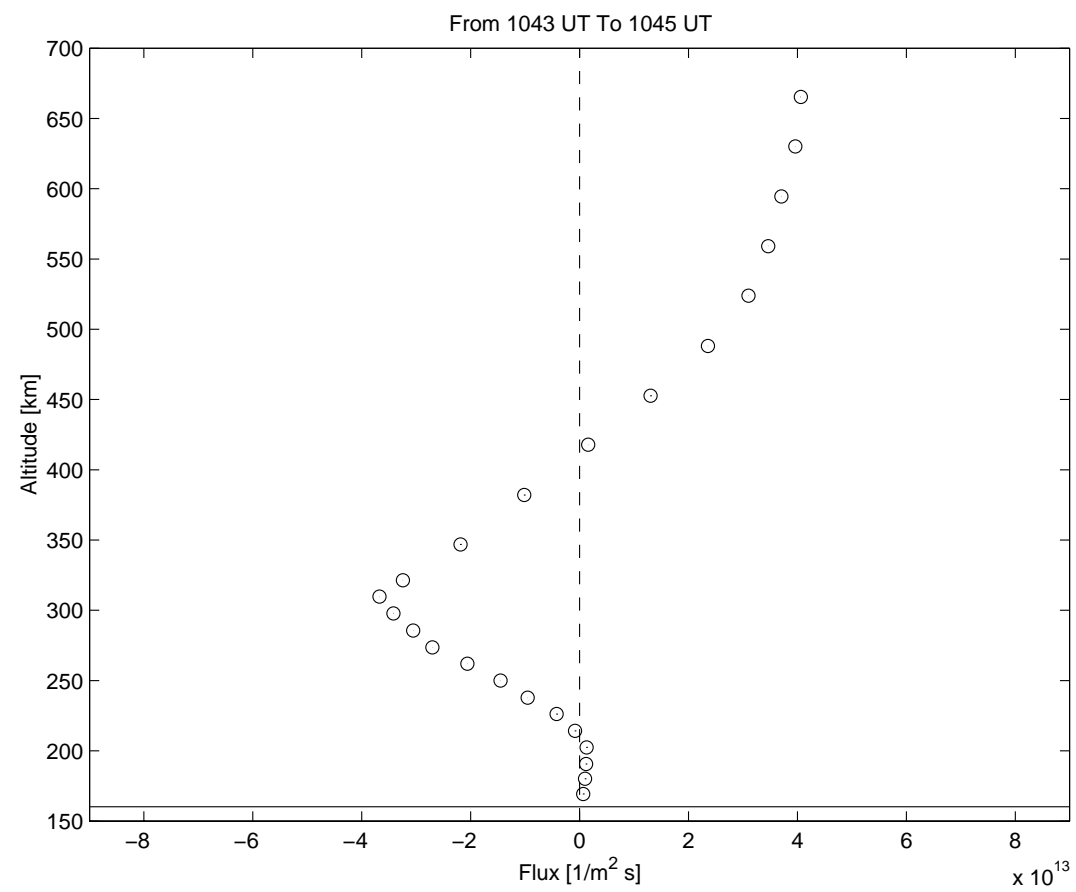

Fig. 3. Field-aligned ion flux calculated from the observed electron density and ion velocity on 08 July 1999, from 10:43 until 10:45 UT. 


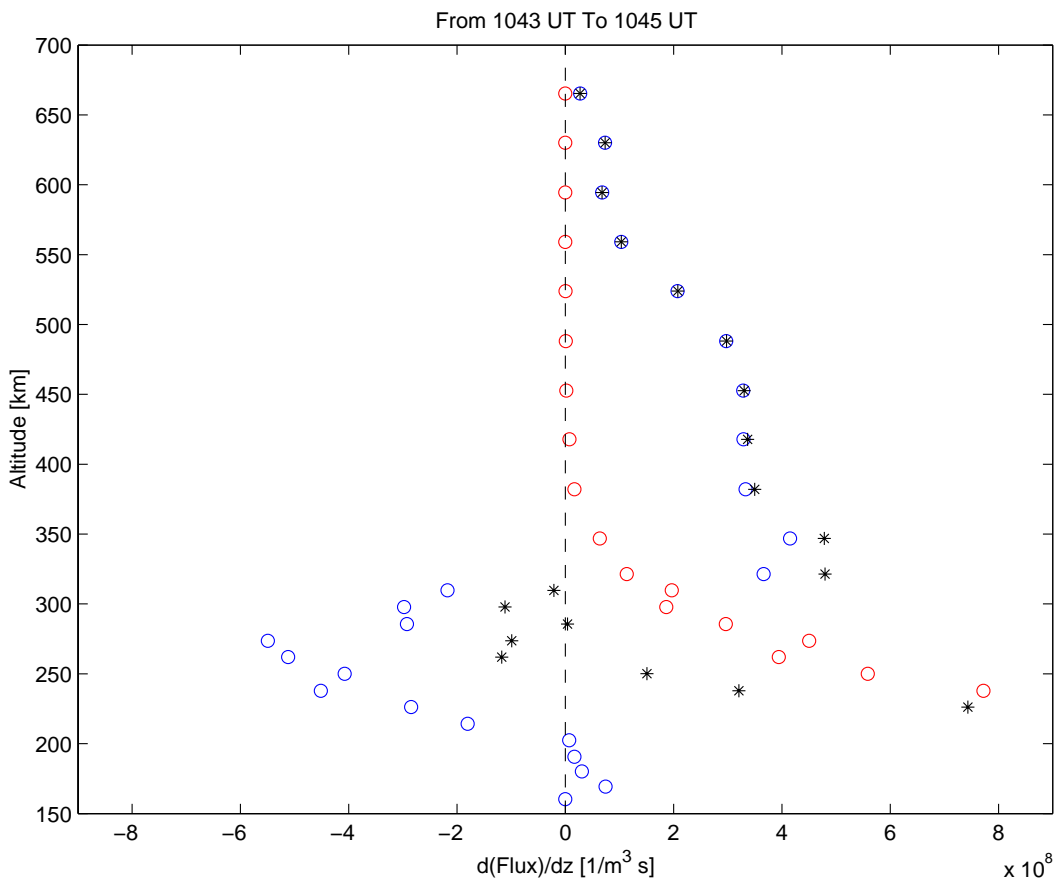

Fig. 4. Profiles of the flux gradient $\partial F_{i} / \partial s$ (blue circles), the negative estimated recombination rate $-L$ (red circles) and the resulting production rate $Q=\partial F_{i} / \partial s+L$ (asterisk) on 8 July 1999, from 10:43 until 10:45 UT.

model (Hedin, 1991), and published values of the reaction rates (Rees, 1989, pp. 278-281). Assuming that Eq. (2) is a good approximation, $Q$ is estimated simply according to $Q=\partial F_{i} / \partial s-L$. Figure 4, which is based on a combination of observations and models, shows that

- below about $200 \mathrm{~km}$ transport is negligible for the ionization balance;

- above about $310 \mathrm{~km}$ ionization is mainly removed by field-aligned transport;

- between about 200 and $310 \mathrm{~km}$ both transport and recombination are important.

Above about $500 \mathrm{~km} \partial F_{i} / \partial s \approx 0$. There seems to be little ionization, recombination is very slow, and consequently, the flux is approximately constant. This is achieved by upward flow velocity that increases with altitude, while $N_{e}$ decreases, similar to a "polar wind."

We have chosen to show profiles from 8 July 1999 just at 10:43-10:45 UT, since there happens to be a pass of the satellite F14 of the DMSP between 10:48:07 and 10:48:12 UT across $75.2^{\circ}$ north geomagnetic latitude. The footprint of the satellite projected along the geomagnetic field down to $350 \mathrm{~km}$ altitude comes close to the ESR antenna beam and is at a distance of $102 \mathrm{~km}$ further west. The particle detector of F14 sees at this time downward-going electrons with an average energy of about $126 \mathrm{eV}$. The observed integrated energy flux $J_{e}$ is relatively high, about $0.21 \mathrm{~mW} \mathrm{~m}^{-2}$, and the integrated electron number flux is $1.04 \cdot 10^{13} \mathrm{~m}^{-2} \mathrm{~s}^{-1}$ at the altitude of the satellite $(840 \mathrm{~km})$, averaged over the 5-s long time period above.

The effect of soft electron precipitation on the ionosphere has been modeled, among others, by Millward et al. (1999). We, now, pick one altitude, $350 \mathrm{~km}$, and use their Fig. 1 to derive a very simplified relation between the ionization rate at $350 \mathrm{~km}$ height, $Q_{350}$, and the flux of $126 \mathrm{eV}$ electrons, $F_{e, 126 \mathrm{eV}}$ :

$Q_{350} \approx 0.3 \cdot 10^{-4} \mathrm{~m}^{-1} F_{e, 126 \mathrm{eV}}$

Where $F_{e, 126 \mathrm{eV}}$ is in $\mathrm{m}^{-2} \mathrm{~s}^{-1}$, and $Q_{350}$ in $\mathrm{m}^{-3} \mathrm{~s}^{-1}$.

Mapping the $126 \mathrm{eV}$ electron flux from the spectrogram of the DMSP F14 particle detector at $840 \mathrm{~km}$ altitude down to $350 \mathrm{~km}$ and using Eq. (3) we can predict that the ionization rate $Q_{350} \approx 3.810^{8} \mathrm{~m}^{-3} \mathrm{~s}^{-1}$. Figure 4 shows the ionization rate estimated from ESR observations using Eq. (2). The value at $350 \mathrm{~km}$ altitude is about $4.510^{8} \mathrm{~m}^{-3} \mathrm{~s}^{-1}$, in good agreement with the prediction from DMSP data and the model by Millward et al. (1999). We have not taken into account the ionization rate due to solar radiation and due to down-going ions that are also seen by the DMSP particle detector but with a much lower energy flux. The estimated solar ionization rate using the $F_{10.7} \mathrm{~cm}$ flux for 8 July 1999 is about $10^{8} \mathrm{~m}^{-3} \mathrm{~s}^{-1}$ (Rees, 1989, p. 257), clearly less than the estimated ionization rate due to electrons. If there are no losses whatsoever, with an ionization rate of $4.510^{8} \mathrm{~m}^{-3} \mathrm{~s}^{-1}$, it takes about $7 \mathrm{~min}$ to produce the density enhancement at 10:48 UT over the ambient plasma density before 9:50 UT.

It seems that in this case cusp electrons are the dominant ionization source in the F-region around $350 \mathrm{~km}$. In 
summary, a detailed analysis of ESR and conjugate DMSP particle data show that intense soft electron precipitation with energies of a few hundred $\mathrm{eV}$ is associated with diverging field-aligned ion flow in the F-region ionosphere.

Variations of the ion flux as well as of the pressure $p=N_{e} k_{B}\left(T_{i}+T_{e}\right)$ and of the field-aligned pressure gradient offset by gravity are shown in Fig. 5. Also, the pressure has a maximum in the F-region roughly at the altitude where the peak of $N_{e}$ is located. The pressure gradient force pushes the ionospheric plasma upward above the maximum and downward below. This qualitatively explains the observation of the diverging field-aligned flow. However, a more quantitative discussion of the force balance turns out to be difficult. Already Wahlund et al. (1992) showed cases where the forces estimated from IS radar data in Troms $\varnothing$ seem to be unbalanced in the F1-region and have a net downward component. According to our experience, this is rather consistently the case at Troms $\varnothing$ and at Svalbard. The pressure force acts even when the F-layer is produced by solar radiation only, and when there is no significant additional ionization. A possible weak diverging flow should be a common feature of the high-latitude ionosphere. But this actually has not been noticed, particularly not in the IS data from Troms $\varnothing$, and is thus not common. It could be that considerable precipitation flux and corresponding enhancements of the "classical" Flayer are needed in order to drive a diverging flow. We will investigate statistically in the next section conditions when diverging field-aligned flows occur.

Ion flux and the pressure gradient reverse direction from downward to upward at certain altitudes, which, in the top and bottom panels of Fig. 5, respectively, are recognized by layers in the color blue. At first glance we would expect that these altitudes in the top and bottom panels are similar, however, they are different. The pressure gradient in the bottom panel reverses at a relatively constant altitude of about 380 $\mathrm{km}$. The energy of the cusp particles does seem to change, but not so much in all the flux tubes that are drifting through the ESR beam, and we can assume that for this event, particle precipitation does not vary strongly in time for a flux tube drifting with the convection. However, the ion flux/flow in the top panel reverses direction at altitudes varying from about 300 to $500 \mathrm{~km}$. The time scale of the variations of the flux/flow reversal resembles that of typical gravity waves. We have seen even more clearly similarly varying flow reversals in several other events not shown here. Gravity waves seen at Troms $\emptyset$ with IS radar have been observed to have amplitudes of about $4 \mathrm{~ms}^{-1}$, and up to about $10 \mathrm{~ms}^{-1}$ in the field-aligned ion velocity (e.g. Hocke et al., 1996). This is considerably smaller than the velocities observed here. The ion acceleration due to a velocity difference between ion and neutrals of $10 \mathrm{~ms}^{-1}$ amounts to about $8 \mathrm{~ms}^{-2}$ near the Fregion peak, roughly $20 \%$ of the acceleration due to pressure gradients, as shown in Fig. 5.

The diverging field-aligned flows are unlikely to be caused by gravity waves, however, their presence can be significant for the force balance during cusp precipitation. Therefore, it is suggested that drag on the ions by the neutral wind might explain the varying "offset" in height between the reversal of the flow and of the plasma pressure gradient.

We finally discuss aspects of the energy balance in events with simultaneous up- and downflow. Figure 6 shows the contributions from photoelectrons, heat conduction and heat advection to total heat flux for the time where we have discussed already the ionization balance in detail. The photoelectron contribution has been estimated according to Schunk and Nagy (2000) and references therein, as well as the contribution from heat conduction where we have used the observed electron temperature. $T_{e}$ increases with altitude, thus heat is conducted downward at all altitudes. Below about $550 \mathrm{~km}$ the solar energy input is more important than heat conduction. Other studies with IS radars (e.g. Doe et al., 2001) have come to similar results.

Here we have added an estimate of the advected heat flow assuming that field-aligned ion flow is not associated with electric current. Thus, the electrons carry most of the thermal energy flow together with the ions. Then the advected heat flux is given by the terms $\boldsymbol{u}_{e} \cdot \nabla\left(3 / 2 p_{e}\right)+5 / 2 p_{e}\left(\nabla \cdot \boldsymbol{u}_{e}\right)$ in Eq. (11.65) in Schunk and Nagy (2000) with $\boldsymbol{u}_{e}=\boldsymbol{v}_{i}$. From Fig. 6 we can see that heat is advected both downward below the F -layer maximum and upward above. Heat is advected above about $300 \mathrm{~km}$ against the temperature gradient, more or less canceling the heat flow due to thermal conduction. This advected heat flow is, therefore, not negligible in a sunlit ionosphere above about $500 \mathrm{~km}$. In a non-sunlit ionosphere the advected heat flow can be important at all altitudes of the F-region.

The features of the event shown and discussed here are also typical for other events that have been seen with the ESR, as we have convinced ourselves.

\section{Statistical occurrence}

How often and under what conditions does a diverging fieldaligned flow occur? The velocity profiles of Common ESR Programmes in 1998-2000 were scanned for up- and downflow events according to criteria illustrated in Fig. 7. Similar criteria were used and are discussed in Endo et al. (2000) for a statistical study of VHF data, where, however, simultaneous up- and down- (i.e. diverging field-aligned) flow was not searched for in that study. In VHF data it is an uncommon feature, probably because sufficiently intense soft particle precipitation occurs predominantly in the cusp which rarely reaches the latitude of the VHF radar.

Statistical occurrences of phenomena at a single site might change with season, $K_{p}$, IMF, etc., either because the phenomenon itself, here up/downflow, is affected by season, geomagnetic activity, or simply because these parameters control the location where up/downflow occurs. This must be kept in mind when interpreting the results that are discussed below.

Occurrences of simultaneous up- and downflow for ESR are shown in Fig. 8. As might be expected, one is more likely to observe upflow and also downflow during higher 


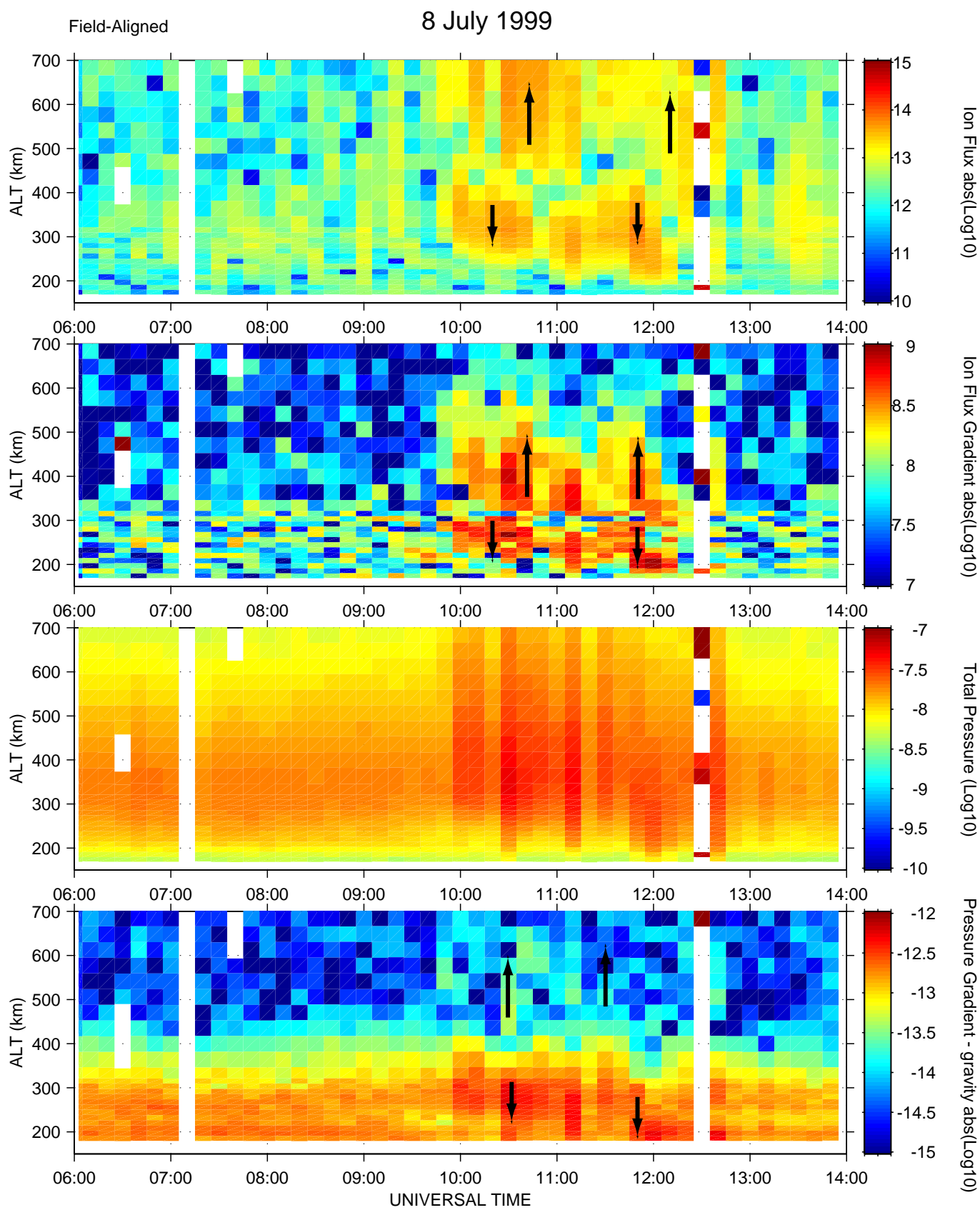

Fig. 5. Shown are parameters derived from the data displayed in Fig. 1. Panels from top to bottom show the absolute value of $F_{i}$, the flux gradient $\partial F_{i} / \partial s$, the total pressure $p=N_{e} k_{B}\left(T_{i}+T_{e}\right)$, and the absolute value of the pressure gradient offset by gravity, $\partial p / \partial s-m_{i} N_{e} g$. The parameters are color-coded over altitude (y-axis) and time (x-axis). The directions of the flux and the pressure gradient in the top and bottom panels, respectively, is indicated by black arrows for the sake of clarity.

geomagnetic activity, according to the $K_{p}$ index. Up- and simultaneous downflow is concentrated within a few hours around magnetic local noon, which is at about 9:30 UT for the ESR location. For $K_{p} \geq 3$ upflow is also relatively common in the evening hours, but rarely accompanied by downflow. We suggest that this evening upflow is related to auroral phenomena on the nightside, while the upflow around local magnetic noon is a feature that is commonly observed around the ionospheric projections of the cusp.
In data from the DMSP F7 and F9 satellites the cusp has been identified as a region of direct magnetosheath plasma entry with electron energies typically $30 \mathrm{eV}$ to $100 \mathrm{eV}$ and high fluxes (Newell and Meng, 1992). This plasma entry generates electron precipitation and patches of enhanced electron density and elevated electron temperature in the F2region that are seen in IS radar data, as case studies have shown (Watermann et al., 1994b). Our data show that soft particle precipitation associated with the cusp seems to cause 


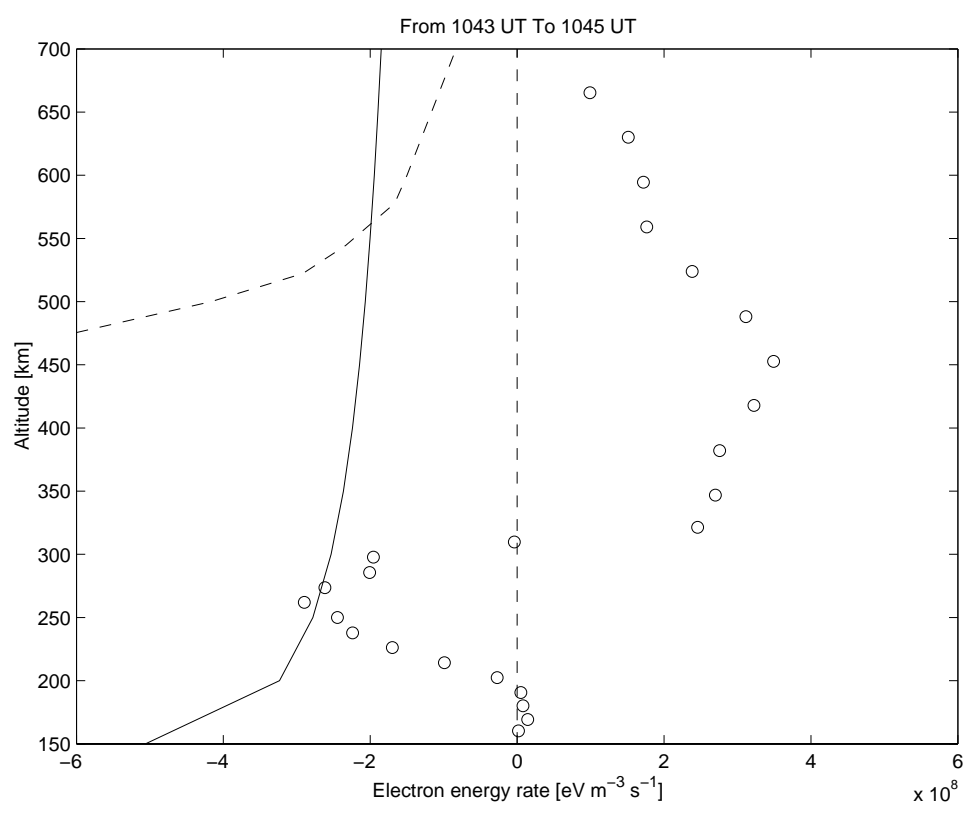

Fig. 6. Contributions from photoelectrons (dashed line), heat conduction (solid line) and advection (circles) to the total heat flux on 199907-08, from 10:43 until 10:45 UT.

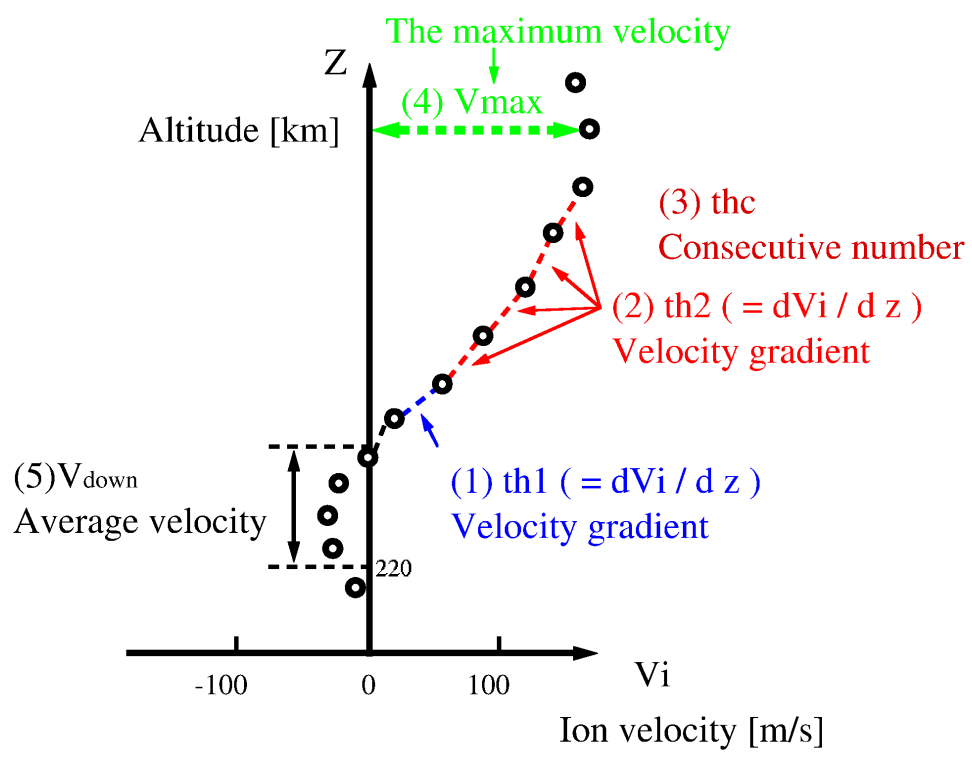

Fig. 7. The criteria for searching velocity profiles automatically for simultaneous up- and downflow events are illustrated. An upflow event is detected if 1) the velocity gradient at the lowest positive velocities exceeds $\left.0.3 \mathrm{~ms}^{-1} \mathrm{~km}^{-1}, 2\right)$ there is an increasing velocity for at least 3 more data points above, with a gradient of at least $0.2 \mathrm{~ms}^{-1} \mathrm{~km}^{-1}$, and the highest positive velocity is at least $200 \mathrm{~ms}$. A simultaneous downflow is detected, if 1 ) there is an upflow event and 2) the average velocity over 4 data points below the upflow is less than $-20 \mathrm{~ms}^{-1}$.

upflow and also the diverging field-aligned flow relatively often, on average, in about $30 \%$ of all cases when upflow occurs.

In winter upflow seems to occur more frequently than in summer. This could be because the average cusp location with respect to the ESR is perhaps more favourable in the winter, but also ion upflow might be more easily generated in a winter ionosphere. However, relatively simultaneous up- and downflow is more common in summer. Then both solar radiation and soft particle precipitation combined can produce an F-layer of high density with corresponding large up- and downward pressure gradients, which, as we suggest, explains this finding.

If diverging field-aligned flow is a typical cusp phenomenon and produced mainly by direct magnetosheath plasma entry, then the direction of the interplanetary magnetic field IMF should have a strong influence on the occurrence frequency. This is confirmed in Fig. 9, where 

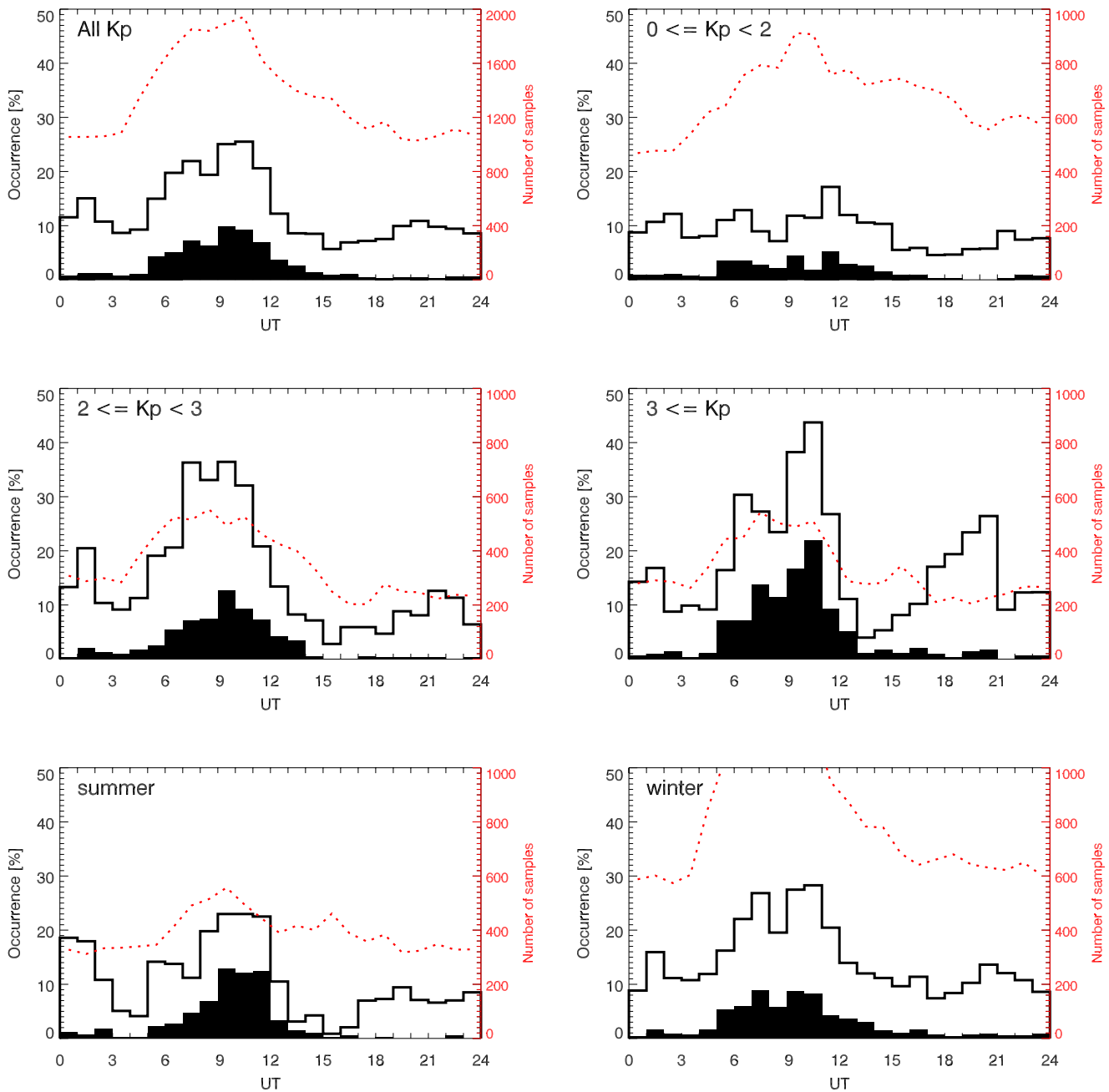

Fig. 8. Histograms of occurrence frequency of up- and downflow over UT for different geomagnetic activity levels according to the $K_{p}$ index and for different seasons. The outlined histogram indicates the occurrence frequency of upflow events and the histogram filled with black indicates how often simultaneously downflow occurred. Also, the number of available velocity profiles is displayed (red dotted lines).

the occurrence for different directions of IMF and different ranges of solar wind pressure is shown. Generally we find that the dependence of the cusp location on the IMF, as it is indicated by ion upflow in our data, is consistent with previous results based on satellite data, e.g. by Newell et al. (1989) and by Aparicio et al. (1991). A negative $\mathrm{z}$ component of IMF enhances greatly the chance to observe up/downflow, moving the cusp to the latitude of the ESR. In these periods a negative y component of IMF enhances the chances to see up/downflow after magnetic local noon, compared to the periods with a positive y component, in agreement with the expected trend for the location of the cusp (Candidi et al., 1989).

Also, the solar wind kinetic pressure $p$ has been found to dramatically affect the map of magnetospheric projections and ionospheric cusp area (Newell and Meng, 1994). In our data shown in Fig. 9, a more frequent, and in local time a more widespread occurrence of ion up- and downflow is seen for $p>2 \mathrm{nPa}$ than for $p<2 \mathrm{nPa}$.

\section{Summary and Conclusions}

A new type of field-aligned plasma flow, diverging from roughly the electron density peak in the F2-region, is seen to occur relatively often in the ionosphere above the ESR. Flow upward from the peak normally continues up to and beyond the highest altitudes seen by the radar, around $700 \mathrm{~km}$ and above. Flow downward from the peak decreases again in the F1-region, where recombination becomes sufficiently fast and eats up the down-going flux.

Diverging field-aligned flow is seen predominantly when soft particle precipitation enhances both the F2-layer and the electron temperature strongly above the values of the ambient plasma. Plasma is transported away from a layer of strong ionization that is generated by a combination of precipitation and solar radiation. In these events the transport exceeds greatly in efficiency recombination, and ensures that not even higher F2 densities are seen. Vertical transport should influence strongly the ion composition. The dominant 

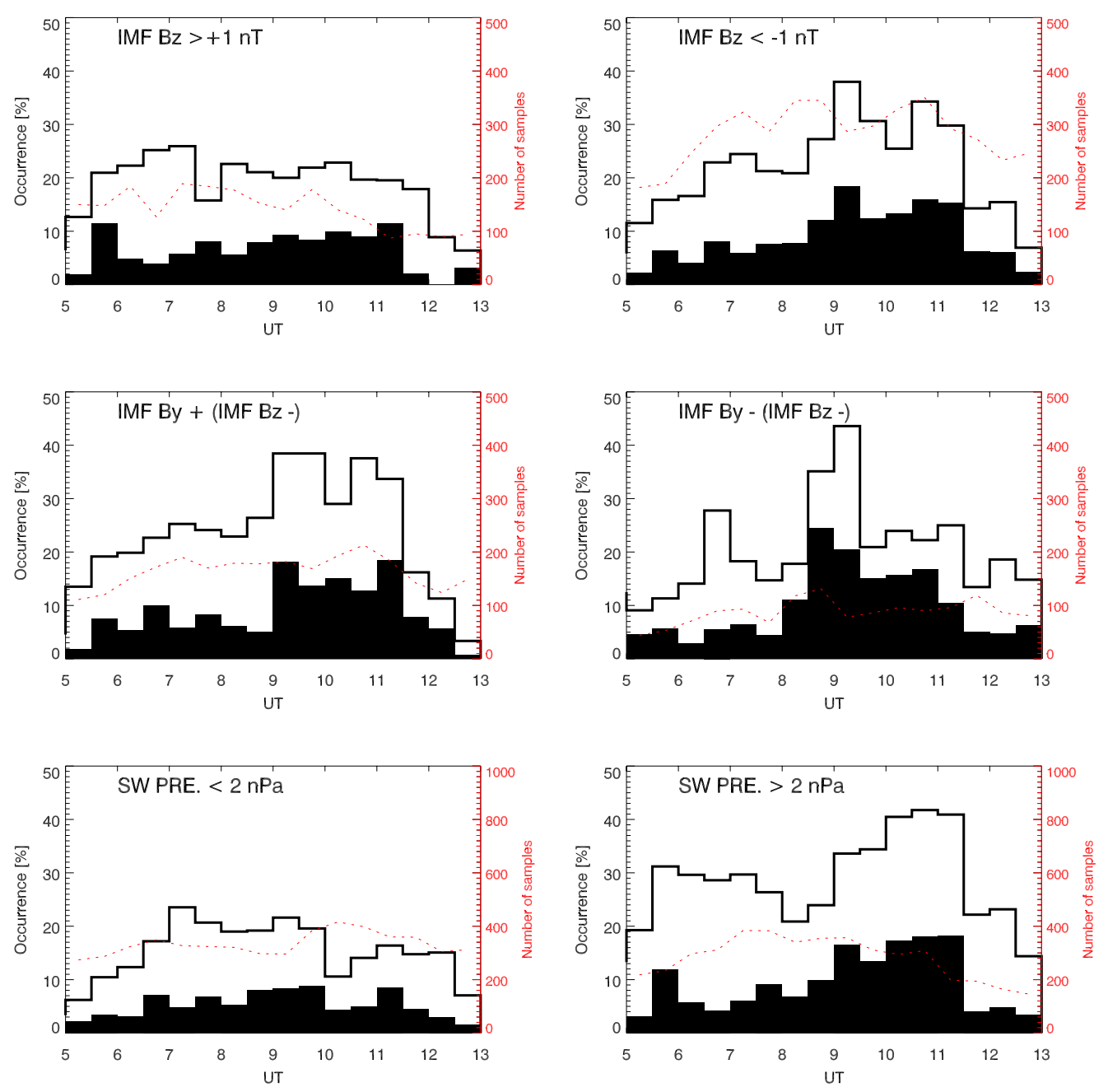

Fig. 9. Histograms of occurrence frequency of up- and downflow over UT for different directions of the interplanetary magnetic field and for different solar wind pressures. The outlined histogram indicates the occurrence frequency of upflow events and the histogram filled with black indicates how often simultaneously downflow occurred. Also, the number of available velocity profiles is displayed (red lines).

ion produced at the density peak is $\mathrm{O}^{+}$which then gets transported both downward and upward. Therefore, we expect that the transition from molecular ions to $O^{+}$is at a lower altitude compared to situations without diverging flow. IS radar cannot distinguish wellbetween molecular ions and atomic oxygen, and we are not able to confirm this expectation with our data. The fitted ion temperatures in the F1-region could be effected by deviations of the ion composition from the model used in our analysis. At the same time, we expect that the transition from $\mathrm{O}^{+}$to a plasma dominated by $\mathrm{H}^{+}$is shifted toward higher altitudes in regions of ion upflow. This transition height is most likely beyond the reach of the ESR facility.

ISR data do not reveal the histories of observed convecting field lines, but only snapshots and thus have limitations. We have studied the direction of the force balance along the field line and found that flow and force directions sometimes don't agree. The drag from neutral wind is suggested to play a role in the field-aligned force balance. This could explain why color plots of field-aligned flow sometimes seem to show a modulation resembling that of gravity waves.

We have drawn attention to the heat that is advected by upand downward plasma flow. An estimate has shown that the advected heat flux is comparable in magnitude to the conducted heat. Thus, ion upflow can decrease or cancel heat conduction from space to the ionosphere. This subject should be studied more systematically in the future.

The flow is diverging along the field line in roughly $30 \%$ of all cases of ion upflow above the ESR on the dayside. Nightside upflow, which is probably connected with the auroral zone, is rarely accompanied by a downflow on the same field line. We suggest that typically only direct entry of sheath plasma and the cusp provide sufficiently intense particle precipitation. This and the possible additional solar radiation are the conditions favoring the occurrence of diverging flow. Consequently, the interplanetary magnetic field and to 
a lesser extent, also the solar wind pressure influence how likely diverging flow occurs.

Diverging field-aligned flow in the polar ionosphere is another of the many links in solar-terrestrial relations. It has been demonstrated in many works how the interplanetary magnetic field controls plasma convection in the magnetosphere and high-latitude ionosphere. Here we have shown that field-aligned transport is also a part of the dynamics in the polar ionosphere that is controlled by the IMF and the solar wind.

To our knowledge models have been able to reproduce frequently observed ion upflow in the high latitude ionosphere, but not the simultaneous up- and downflow reported here. We think that a realistic model when driven by sufficiently intense downward electron fluxes should reproduce the phenomenon.

Acknowledgements. We are indebted to the Director and staff of EISCAT for operating the facility and supplying the data. EISCAT is an International Association supported by Finland (SA), France (CNRS), the Federal Republic of Germany (MPG), Japan (NIPR), Norway (NFR), Sweden (VR) and the United Kingdom (PPARC).

The DMSP particle detectors were designed by Dave Hardy of AFRL, and data obtained from JHU/APL. We also thank D. Hardy, F. Rich, and P. Newell for their use.

\section{References}

Aparicio, B., Thelin, B. and Lundin, R.: The polar cusp from a particle point of view - A statistical study based on viking data, J. Geophys. Res., 96, 14 023, 1991.

Candidi, M., Mastrantonio, G., Orsini, S., and Meng, C.-I.: Evidence of the influence of the interplanetary magnetic field azimuthal component on polar cusp configuration, J. Geophys. Res., 94, 13 585-13 591, 1989.

Chappell, C. R., Moore, T. E., and Waite, J. H.: The ionosphere as a fully adequate source of plasma for the earth's magnetosphere, J. Geophys. Res., 92, 5896-5910, 1987.

Chappell, C. R., Giles, B. L., Moore, T. E., Delcourt, D. C., Craven, P. D., and Chandler, M. O.: The adequacy of the ionospheric source in supplying magnetospheric plasma, J. Atmos. Terr. Phys., 62, 421-436, 2000.

Doe, R. A., Kelly, J. D., and Sánchez, E. R.: Observations of persistent dayside $\mathrm{F}$ region electron temperature enhancements associated with soft magnetosheath-like precipitation, J. Geophys. Res., 106, 3615-3630, 2001.

Endo, M., Fujii, R., Ogawa, Y., Buchert, S. C., Nozawa, S., Watanabe, S., and Yoshida, N.: Ion outflow and inflow at the topside ionosphere observed by the EISCAT VHF radar, Ann. Geophys., 18, 170-181, 2000.

Hagfors, T.: Plasma fluctuations excited by charged particle motion and their detection by weak scattering of radio waves, in: Incoherent scatter, theory, practice and science, edited by Alcaydé, D., EISCAT Technical Report, 1997.

Hedin, A. E.: Extension of the MSIS thermosphere model into the middle and lower atmosphere, J. Geophys. Res., 96, 1159-1172, 1991.
Hocke, K., Schlegel, K., and Kirchengast, G.: Phases and amplitudes of TIDs in the high latitude F-region observed by EISCAT, J. Atmos. Terr. Phys., 58, 245-255, 1996.

Lehtinen, M. and Huuskonen, A.: General incoherent scatter analysis and GUISDAP, J. Atmos. Terr. Phys., 58, 435-452, 1996.

Loranc, M., and St.-Maurice, J.-P.: A time-dependent gyro-kinetic model of thermal ion upflows in the high-latitude $\mathrm{F}$ region, J. Geophys. Res., 99, 17 429-17452, 1994.

Millward, G. H., Moffett, R. J., Balmforth, H. F., and Rodger, A. S.: Modeling the ionospheric effects of ion and electron precipitation in the cusp, J. Geophys. Res., 104, 24 603-24 612, 1999.

Newell, P. T., Meng, C., Sibeck, D. G., and Lepping, R.: Some lowaltitude cusp dependencies on the interplanetary magnetic field, J. Geophys. Res., J. Geophys. Res., 94, 8921-8927, 1989.

Newell, P. T. and Meng, C.: Ionospheric projections of magnetospheric regions under low and high solar wind pressure conditions, J. Geophys. Res., 99, 273-286, 1994.

Newell, P. T. and Meng, C.: Mapping the dayside ionosphere to the magnetosphere according to particle precipitation characteristics, Geophys. Res. Lett., 19, 609-612, 1992.

Nilsson, H., Kirkwood, S., Eliasson, L., Norberg, O., Clemmons, J., and Boehm, M.: The ionospheric signature of the cusp: A case study using freja and the sondrestrom radar, Geophys. Res. Lett., 21, 1923-1926, 1994.

Ogawa, Y., Fujii R., Buchert, S., Nozawa, S., Watanabe, S., and van Eyken, A. P.: Simultaneous EISCAT svalbard and VHF radar observations of ion upflows at different aspect angles, Geophys. Res. Lett., 27, 81-84, 2000.

Rees, M. H.: Physics and chemistry of the upper atmosphere, Cambridge University Press, New York, 1989.

Rietveld, M. T., Collis, P. N., and St.-Maurice, J.-P.: Naturally enhanced ion acoustic waves in the auroral ionosphere observed with the EISCAT 933-MHz radar, J. Geophys. Res., 96, 19291, 1991.

Schunk, R. W. and Nagy, A. F.: Ionospheres, Cambridge University Press, New York, 2000.

Wahlund, J.-E., Opgenoorth, H. J., Häggström, I., Winser, K. J. and Jones, G. O. L.: EISCAT observations of topside ionospheric ion outflows during auroral activity - revisited, J. Geophys. Res., 97, 3019-3037, 1992.

Wannberg, G., Wolf, I., Vanhainen, L.-G., Koskenniemi, K., Röttger, J., Postila, M., Markkanen, J., Jacobsen, R., Stenberg, A., Larsen, R., Eliassen, S., Heck, S., and Huuskonen, A.: The EISCAT Svalbard radar: A case study in modern incoherent scatter radar system design, Radio Sci., 32, 2283-2307, 1997.

Watermann, J., de la Beaujardiere, O., and Newell, P. T.: Incoherent scatter radar observations of ionospheric signatures of cusp-like electron precipitation, J. Geomag. Geoelectr., 44, 1195-1206, 1992.

Watermann, J., de La Beaujardiere, O.,Lummerzheim, D., Woch, J., Newell, P. T., Potemra, T. A., Rich, F. J., and Shapshak, M.: The dynamic cusp at low altitudes: A case study, utilizing viking, DMSP-F7 and Sondrestrom incoherent scatter radar observations, Ann. Geophys., 12, 1144-1157, 1994a.

Watermann, J., Lummerzheim, D., de La Beaujardiere, O., Newell, P. T. and Rich, F. J.: Ionospheric footprint of magnetosheath-like particle precipitation observed by an incoherent scatter radar, J. Geophys. Res., 99, 3855-3867, 1994b. 